Check for updates

Cite this: RSC Adv., 2017, 7, 29606

Received 16th May 2017

Accepted 22nd May 2017

DOI: 10.1039/c7ra05534j

rsc.li/rsc-advances

\section{Controlled transportation of droplets and higher fog collection efficiency on a multi-scale and multi-gradient copper wire $\uparrow$}

\author{
Yan Xing, $\$$ Sijie Wang, $\$$ Shile Feng, Weifeng Shang, Siyan Deng, Lei Wang, (10 \\ Yongping Hou (D)* and Yongmei Zheng*
}

Via a one-step gradient anodic oxidation, a copper wire with a multi-scale structure and multi-gradient was successfully fabricated, which could realize self-propulsion of droplets and with a well-controlled transport distance. Compared to the previous study, herein, the wire exhibited not only a longer transport distance of the droplet, but also a much higher water collection efficiency.
Surfaces in possession of transporting droplet have attracted significant attention because of their promising application in advanced materials. ${ }^{1}$ Inspired by the special directional transport properties of creatures in nature including those of butterfly wings and rice leaves, ${ }^{2}$ several possible methods have been developed to control the movement behaviour of droplets. ${ }^{3}$ Droplet transport on a one-dimensional surface has extensive application in the domain of fog-water collection, ${ }^{4}$ droplet condensation, ${ }^{5}$ dehumidification and high-efficiency humidity control; ${ }^{6}$ therefore, some related studies have been conducted and the droplet transport on a one-dimensional surface has been realized in some cases. ${ }^{7}$ Quéré et al. have presented that condensing water droplets can be self-removed from the legs of water striders during the condensation process owing to the elastic deformation of the network of setae. ${ }^{8}$ Lorenceau et al. demonstrated that a drop of a wetting liquid placed on a conical fibre spontaneously moved towards the regions of large radius under the effect of Laplace pressure gradient. ${ }^{9}$ Zheng et al. reported that a substrate curvature gradient can accelerate micro-and nanodroplets to high speeds on a tapered glass tube with less contact angle hysteresis (CAH) $\left(1.5-3.7^{\circ}\right) .{ }^{10}$ Actually, the surface of the wire (or fiber) usually has great roughness and $\mathrm{CAH}$, which would suppress the movement of the droplet in most cases. ${ }^{11}$ To the best of our knowledge, it is a great challenge to achieve controlled self-propulsion of a droplet on a partial wetting one-dimensional surface in a normal environment (not high humidity) via a simple method..$^{12,13}$

Key Laboratory of Bio-Inspired Smart Interfacial Science and Technology of Ministry of Education, School of Chemistry and Environment, Beihang University, Beijing, 100191, P. R. China.E-mail: houyongping09@buaa.edu.cn; zhengym@buaa.edu.cn

$\dagger$ Electronic supplementary information (ESI) available: Fig. S1-S5, Table S1. Experimental apparatus, XPS results, oil droplet movement, the change of ACA, CA and RCA on Sample-A. See DOI: 10.1039/c7ra05534j

$\$$ These authors contributed equally to this study and share first authorship.
Herein, we used a one-step gradient anodic oxidation to fabricate the gradients of a multi-scale structure, chemical composition and Laplace pressure on a copper $(\mathrm{Cu})$ wire to realize self-propulsion of a droplet. More importantly, transportation distances could be controlled and predicted very well. The results indicate that compared to the previous study, ${ }^{13}$ the copper wire exhibits not only a longer transportation distance of the droplet, but also a much higher water collection efficiency. This study is significant for designing materials for developing applications in fog-water collection, ${ }^{14}$ droplet transportation, ${ }^{15}$ dehumidification, ${ }^{16}$ droplet condensation, ${ }^{17}$ etc.

To fabricate a wettable gradient on the surface of a copper wire, we employed the gradient anodic oxidation method to form the gradients in roughness and chemical composition (Fig. 1a). ${ }^{12}$ The oxidation extent on different areas of the copper wire could be controlled via current and oxidation time gradient. In Fig. 1b, the Scanning Electron Microscopy (SEM) images show the structure gradient of Sample-A (current: 0.5 A, volume flow velocity of electrolyte: $13.2 \mathrm{~L} \mathrm{~h}^{-1}$ ). At the top part, the surface is relatively smooth (Fig. 1b0). Along the direction from $\mathrm{T}$ to $\mathrm{B}$ ( $\mathrm{T}$ : top area and B: bottom area of the copper wire during gradient anodic oxidation), cracks arise (Fig. $1 \mathrm{~b} 1$ and 1b2) and then transform into cavernous structures (Fig. 1b3). Finally, some different-sized crisp and porous structures appear (Fig. 1b and b3-b5) and the multi-scale structure is formed. In addition, we also observe a small change in the diameter of the copper wire after different gradient anodic oxidation treatments. The diameter changes from $150 \mu \mathrm{m}$ to $230 \mu \mathrm{m}$ (corresponding to a cone with an apex angle $(\alpha)$ of $1.6^{\circ}$, as shown in Fig. 1b) along the direction from $\mathrm{T}$ to $\mathrm{B}$ because of the formation of a loose structure. Accordingly, the SEM results indicate that not only the multi-scale and conical structure but also a roughness gradient are formed via the one-step gradient anodic oxidation method. 


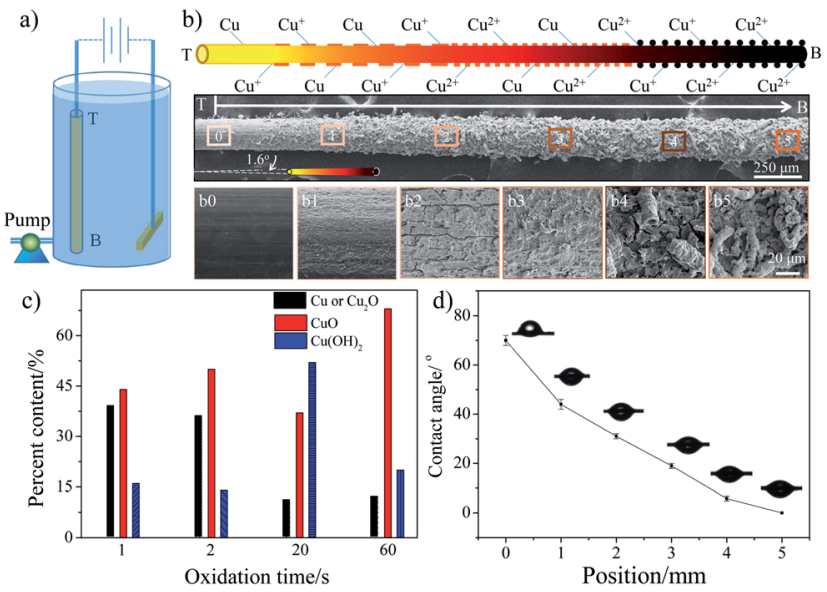

Fig. 1 Schematic of the formation of wettable gradient and Laplace pressure gradient on a copper wire and the change in the microstructure, chemical composition, and water contact angle on SampleA surface. (a) Apparatus for gradient anodic oxidation. (b) Schematic of the change in microstructure, chemical composition and SEM images of the copper wire surface (Sample-A). SEM images show that crisp and porous structures gradually appear after applying the gradient anodic oxidation process and the obvious directional roughness gradient is formed. In addition, the change in the diameter of the wire is also observed and a conical structure is formed (T: top area and B: bottom area of the copper wire during gradient anodic oxidation). (c) Chemical composition of copper wire prepared at different anodic oxidation time. The chemical composition gradient is also formed during the gradient anodic oxidation process. (d) Contact angles of Sample-A at different areas (the zero position is defined as the boundary between unoxidized part and oxidized part).

The chemical properties of the copper wire were analysed using X-ray photoelectron spectroscopy (XPS). Fig. S1a $\uparrow$ represents the XPS spectrum of $\mathrm{Cu}(2 \mathrm{p})$ for copper wires after different oxidation times. The presence of shake-up satellite peaks confirms the formation of $\mathrm{Cu}^{2+}$ on the surface, i.e., cupric oxide $(\mathrm{CuO})$ or cupric hydroxide $\left(\mathrm{Cu}(\mathrm{OH})_{2}\right) \cdot{ }^{14,15}$ By investigating the binding energy for each core level, one can identify the detailed surface chemical composition. ${ }^{16}$ Because $\mathrm{Cu} 2 \mathrm{p}_{3 / 2}$ and $\mathrm{Cu} 2 \mathrm{p}_{1 / 2}$ play similar roles in determining the $\mathrm{Cu}$ valence, here only $\mathrm{Cu} 2 \mathrm{p}_{3 / 2}$ is discussed (Fig. S1b, ESI $\dagger$ ). According to the literature, ${ }^{15}$ the $\mathrm{Cu} 2 \mathrm{p}_{3 / 2}$ core level spectrum shows a main peak envelope curve-fitted into three components centered at 932.5, 933.8 and $935.1 \mathrm{eV}$. The peak at $932.5 \mathrm{eV}$ is attributed to either metallic $\mathrm{Cu}$ or $\mathrm{Cu}_{2} \mathrm{O}$. The component at 933.8 and $935.1 \mathrm{eV}$ arises because of the presence of $\mathrm{CuO}$ and $\mathrm{Cu}(\mathrm{OH})_{2}$. The XPS results indicate that with the increase in the oxidation time, the metallic copper or $\mathrm{Cu}_{2} \mathrm{O}$ content decrease obviously, but the $\mathrm{CuO}$ and $\mathrm{Cu}(\mathrm{OH})_{2}$ content increases sharply (Fig. 1c). In other words, the $\mathrm{Cu}$ or $\mathrm{Cu}_{2} \mathrm{O}$ is oxidized to form $\mathrm{Cu}^{2+}$. We also use Energy Dispersive Spectrometry (EDS) to investigate the elements in the copper wire. The EDS analysis results (Table $\mathrm{S} 1 \dagger)$ show that two main elements, copper and oxygen, exist on the copper wire surface. With the increase in the oxidation time, the atomic ratio $(\mathrm{O} / \mathrm{Cu})$ increases from 0.13 to 2.35 , which is consistent with the XPS results. Therefore, the XPS and EDS results suggest that chemical valence $\mathrm{Cu}^{+}$coexists with valence $\mathrm{Cu}^{2+}$. With the increase in the oxidation time, more and more
$\mathrm{Cu}$ or $\mathrm{Cu}^{+}$are oxidized to form $\mathrm{Cu}^{2+}$. Obviously, a chemical composition gradient is also induced on the copper wire during gradient anodic oxidation treatment.

Fig. 1d shows the contact angle (CA) of Sample-A on different areas, which indicates the CA changes gradually from $70^{\circ}$ to $0^{\circ}$ along the direction from $\mathrm{T}$ to $\mathrm{B}$, showing an average wettable gradient of $14^{\circ} \mathrm{mm}^{-1}$ (average wettable gradient: ratio of CA change to the length of wettable gradient). The CA is expected to depend upon the chemical composition and surface morphology. As the distance from the top to the bottom increases, the surface becomes rough gradually (multi-scale structure is formed) and more and more $\mathrm{Cu}$ or $\mathrm{Cu}^{+}$are oxidized to form more hydrophilic $\mathrm{CuO}$ and $\mathrm{Cu}(\mathrm{OH})_{2} \cdot{ }^{17}$ At the bottom part, the surface has a more hydrophilic chemical composition and a rougher structure, resulting in a CA of $0^{\circ}$. Clearly, the formation of a great wettable gradient is mainly ascribed to the cooperation between the chemical composition and the surface morphology (Wenzel's theory ${ }^{18}$ ). To obtain the different wettable gradients, we adjusted the volume flow velocity of the electrolyte to control the oxidation time gradient. The results indicate that the average wettable gradient could be controlled from $35^{\circ} \mathrm{mm}^{-1}$ to $4^{\circ} \mathrm{mm}^{-1}$ via the volume flow velocity of the electrolyte (from $5.3 \mathrm{~L} \mathrm{~h}^{-1}$ to $42.2 \mathrm{~L} \mathrm{~h}^{-1}$ ) and the corresponding length of the wettable gradient changes from 2 to $18 \mathrm{~mm}$ (see Fig. S2 and S3, ESI $\dagger$ ), whereas, the apex angle of the copper wire is always about $1.6^{\circ}$ at different oxidation treatment conditions. Obviously, via the gradient anodic oxidation method, not only a wettable gradient but a Laplace pressure gradient is also induced effectively on the surface of the copper wire and the value of the wettable gradient can be adjusted easily.

The movement behaviours of the droplet on the multigradient surface are observed via a charge coupled device (CCD) camera (Fig. 2a-c). When a water droplet is dripped on the copper wire with a wettable gradient of more than $4.5^{\circ}$ $\mathrm{mm}^{-1}$ (the droplet is dripped on the boundary between the unoxidized part and the oxidized part), it would be driven towards the more hydrophilic area for some distance and then pinned on the copper wire. For different volumes of the droplet,

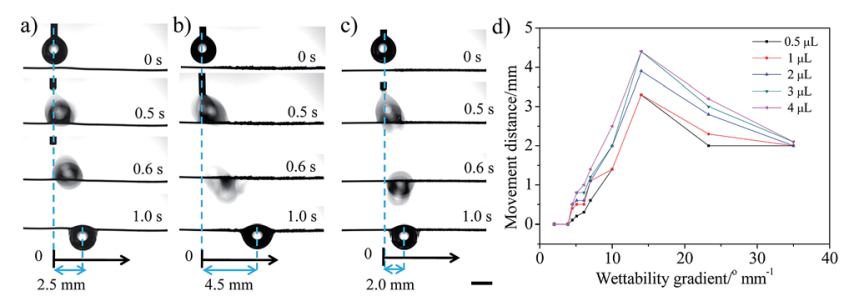

Fig. 2 Water droplet movement behaviour on the copper wire with a disparate wettable gradient (the droplet is dripped on the boundary between the unoxidized part and the oxidized part, $X=0$ ): (a) $10^{\circ}$ $\mathrm{mm}^{-1}$, (b) $14^{\circ} \mathrm{mm}^{-1}$, (c) $35^{\circ} \mathrm{mm}^{-1}$. Optical images show that the water droplet is driven fast towards the more hydrophilic area and shows a different distance with a disparate wettable gradient. The scale is 2 $\mathrm{mm}$. (d) The movement distance of the droplet on the copper wire with different wettable gradients. For different volumes of droplet, the movement distance always increases first and then decreases, showing a peak at $14^{\circ} \mathrm{mm}^{-1}$. 

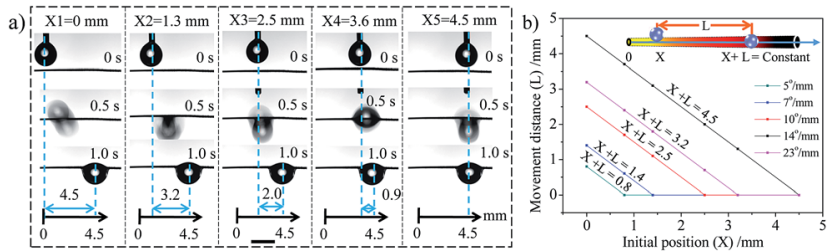

Fig. 3 (a) The droplet movement behaviour on copper wire with a wettable gradient of $14^{\circ} \mathrm{mm}^{-1}$ when the droplet is placed at different initial positions (within balance position). (b) The relationship between the initial position $(X)$ and the movement distance $(L)$ for surfaces with different wettable gradients. There seems to be a balance position and the droplet usually moves to the balance position no matter where the droplet is placed, i.e., $X+L=$ constant ( $X$ is the initial position and $L$ is the movement distance of the droplet. The zero position $(X=0)$ is defined as the boundary between the unoxidized part and the oxidized part). The volume of the droplet is $4 \mu \mathrm{L}$. The scales are $2 \mathrm{~mm}$.

with the increase in the wettable gradient, the movement distance always increases first and then decreases, showing a peak at $14^{\circ} \mathrm{mm}^{-1}$ (Fig. 2d). In addition, an interesting phenomenon is observed that for a given sample, there seems to be a balance position and the droplet usually moves to the balance position no matter where this droplet is placed (within balance position) (Fig. 3a), i.e., $X+L=$ constant ( $X$ is the initial position and $L$ is the movement distance of the droplet. The zero position $(X=0)$ is defined as the boundary between the unoxidized part and the oxidized part). For surfaces with different wettable gradients, the balance position is different (Fig. 3b). For example, for the surface with $14^{\circ} \mathrm{mm}^{-1}$, the balance position is $4.5 \mathrm{~mm}$ (Fig. 3a). For $5^{\circ} \mathrm{mm}^{-1}, 7^{\circ} \mathrm{mm}^{-1}, 10^{\circ}$ $\mathrm{mm}^{-1}, 23^{\circ} \mathrm{mm}^{-1}$, the position changes to $0.8 \mathrm{~mm}, 1.4 \mathrm{~mm}, 2.5$ $\mathrm{mm}, 3.2 \mathrm{~mm}$, respectively (Fig. 3b). However, when a droplet is dripped beyond the balance position, the droplet is always pinned on the wire and no movement is observed. In addition, the copper wire with a wettable gradient could not only drive the water droplet, but also make the oil droplet move on it, and the same behaviour is observed (Fig. S4, ESI†).

In order to understand this unique phenomenon thoroughly, we analysed the forces exerted on the droplets. It is considered that there are three forces that influence the motion of droplets: wettability gradient force $\left(F_{\mathrm{W}}\right)$, Laplace pressure gradient $\left(F_{\mathrm{L}}\right)$ and hysteresis force $\left(F_{\mathrm{H}}\right)$, which can be related to a surface wettability gradient, conical shape of wire and CA hysteresis, respectively. The wettable gradient force induced by diverse CAs on the two sides of the droplet would drive droplets towards the more wettable region of the surface, which could be described as: ${ }^{12}$

$$
F_{\mathrm{W}}=\pi R^{2} \gamma k \sin \theta=F_{\mathrm{W}}(R, k, \theta)
$$

here, $R$ is the base radius of the droplet, $\gamma$ is the surface tension of water, $\theta$ is the position-responsive sessile CA of the droplet and $k$ is the average wettable gradient. The Laplace pressure gradient $\left(F_{\mathrm{L}}\right)$ arises from the curvature gradient of the copper wire, which points to the side with a larger local radii (along the direction from $\mathrm{T}$ to $\mathrm{B}$ ). Megaridis et al. ${ }^{19}$ found the linear nature of the plot of $F_{\mathrm{L}}$ against the apex angle $(\alpha)$. Accordingly, we can evaluate the magnitude of the $F_{\mathrm{L}}$ as:

$$
F_{\mathrm{L}}=A \alpha+B
$$

here, $A, B$ is a constant coefficient for a given volume of the droplet. As the apex angle of the copper wire is about $1.6^{\circ}$ at different oxidation treatment conditions, $F_{\mathrm{L}}$ seemed to be a constant for a given volume of droplet. The hysteresis force $\left(F_{\mathrm{H}}\right)$ is related to the CA hysteresis, opposite to the moving direction, which usually blocks the movement of droplets. The magnitude of $F_{\mathrm{H}}$ is described as: ${ }^{12}$

$$
F_{\mathrm{H}}=2 R \gamma\left(\cos \theta_{\mathrm{r}}-\cos \theta_{\mathrm{a}}\right)
$$

where $\theta_{\mathrm{r}}$ and $\theta_{\mathrm{a}}$ are the position-dependent receding and advancing CAs, respectively. The total force $\left(F_{\mathrm{T}}\right)$ is described as:

$$
\begin{aligned}
F_{\mathrm{T}}= & F_{\mathrm{W}}+F_{\mathrm{L}}-F_{\mathrm{H}}=R \gamma\left(\pi R k \sin \theta-2\left(\cos \theta_{\mathrm{r}}-\cos \theta_{\mathrm{a}}\right)\right) \\
& +F_{\mathrm{L}}=F_{\mathrm{T}}(k, x)
\end{aligned}
$$

Therefore, for a given volume of droplet, $F_{\mathrm{T}}$ is regarded as a function of the average wettable gradient $(k)$ and the position of droplet $(x)\left(\theta, \theta_{\mathrm{r}}\right.$ and $\theta_{\mathrm{a}}$ are only function of position $(x)$ and $F_{\mathrm{L}}$ is a constant). When the value of $k$ is small, the wettable gradient force is small ( $F_{\mathrm{T}}$ is below zero) and no movement of the droplet is observed. The increase in the value of $k$ would induce an increase an $F_{\mathrm{T}}$ (eqn (4)). When the value of $k$ is above some critical value $\left(4.5^{\circ} \mathrm{mm}^{-1}\right.$, Fig. 2$)$, the sum of $F_{\mathrm{W}}$ and $F_{\mathrm{L}}$ surpasses $F_{\mathrm{H}}\left(F_{\mathrm{T}}>0\right)$ and the droplet would be driven to the more wettable side. As the droplet moves to a more wettable area ( $\theta$ decreases), the value of $F_{\mathrm{W}}$ decreases (eqn (1)) and the value of $F_{\mathrm{H}}$ increases gradually (the difference between the $\theta_{\mathrm{r}}$ and $\theta_{\mathrm{a}}$ increases, Fig. S5, ESI $\dagger$ ), which would lead to a decrease in $F_{\mathrm{T}}$. When $F_{\mathrm{T}}=0$, the motion would be halted. Although the increase in $k$ could induce a larger $F_{\mathrm{T}}$, the increase also shortens the length of the wettable gradient gradually, which would limit the driving distance (Fig. S3, ESI $†$ ). Thus, the driving distance shows a peak at $14^{\circ} \mathrm{mm}^{-1}$ (the surface has larger $F_{\mathrm{T}}$ and length of wettable gradient). It seems that when $k<14^{\circ} \mathrm{mm}^{-1}$, the movement distance is mainly controlled by the wettable gradient and the Laplace pressure gradient. When $k>14^{\circ}$ $\mathrm{mm}^{-1}$, the movement distance is mainly controlled by the length of the wettable gradient. For example, for $23^{\circ} \mathrm{mm}^{-1}$, the movement distance $(3.2 \mathrm{~mm})$ is very close to the length of the wettable gradient $(3.1 \mathrm{~mm}$, Fig. $\mathrm{S} 3 \uparrow)$, consistent with the speculation above. For a given surface ( $k$ is a constant), $F_{\mathrm{T}}$ could be regarded as a function of position $(x)$, i.e., $F_{\mathrm{T}}(x)$. Accordingly, the droplets would always stop and be balanced at the same position $\left(F_{\mathrm{T}}(x)=0\right)$ no matter where the droplets were released (within the balance position), i.e., $X+L=$ constant, which corresponds well with our experimental results (Fig. 3b). Obviously, the result implies that the movement behaviors of the droplet could be predicted and controlled by the initial position and/or the magnitude of the wettable gradient (according to Fig. $3 b)$.

More importantly, the introduction of surface wettability and the Laplace pressure gradient also helps the directional 
transformation of the water drop during the fog-water collection process, which in turn speeds up the drop coalescence and improves the water collection efficiency. The comparison of the fog-water collection behaviour between the original copper wire and Sample-A is shown in Fig. $4 \mathrm{a}$ and b. The results indicate that on the original copper wire, the droplets simply grow up at the initial position during the collection process. As for SampleA, the droplets move towards to the more hydrophilic area and gradually coalesce into a bigger one during the collection process. Compared to the recent research with a transportation distance of $5 \mathrm{~mm},{ }^{13}$ the water droplet could achieve a much longer transportation length of $\sim 1 \mathrm{~cm}$ in our study, which means that the transportation distance is further increased nearly 1 time. Ju et al. ${ }^{20}$ got a one period collection process on CCW with a gradient wettability in $56.9 \mathrm{~s}$ with a fog flow velocity of about $20-30 \mathrm{~cm} \mathrm{~s}^{-1}$ at room temperature, while the transport distance was only $823 \mu \mathrm{m}$. Heng et al. ${ }^{21}$ developed a branched $\mathrm{ZnO}$ wire structure for water collection, and collected $6 \mu \mathrm{L}$ of water within $30 \mathrm{~min}$. Xu et al. ${ }^{22}$ designed a water collecting array of PCCWs, and when they put this array at a room temperature of $15{ }^{\circ} \mathrm{C}, 90 \%$ relative humidity, and a fog impacting velocity of $2.4 \mathrm{~m} \mathrm{~s}^{-1}$ with an impact angle of $90^{\circ}$, the highest water collection rate can reach $0.618 \mathrm{~g} \mathrm{~cm}^{-2} \mathrm{~h}^{-1}$. Different researchers investigated their samples in different systems, which makes it difficult to compare the results with our results absolutely. Therefore, we compared Sample-A to the original copper wire in the same system. Fig. 4c shows a comparison of the water hanging ability and water collection efficiency between the original copper wire and Sample-A. The maximal water-hanging volume of Sample-A exceeds by $12.84 \mu \mathrm{L}$, i.e., increases by $25 \%$, which may account for the roughness of the structures. Meanwhile, the time for one period collection process before the hanging droplet detaches from the wire shortens $(64 \mathrm{~s}$, decreases by $30 \%$ ) and the water collection efficiency increases by $78 \%$. Obviously, after imposing a gradient anodic oxidation
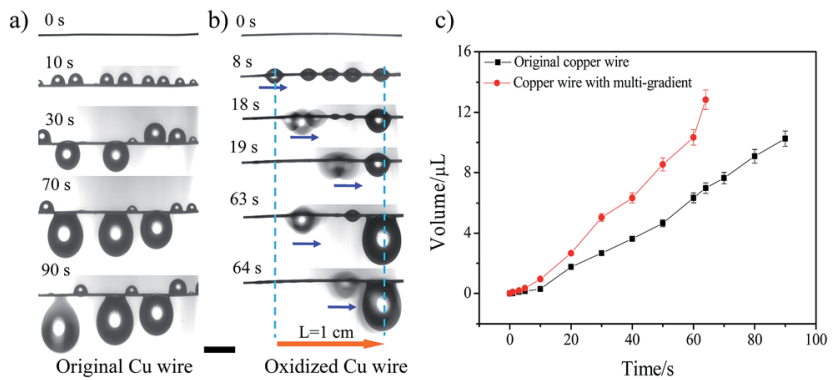

Fig. 4 Comparison of fog-water collection behavior between original copper wire and multi-gradient copper wire. (a) Water collection process of original copper wire. (b) Water movement behavior on the surface of Sample-A. The results indicate that the droplets simply grow at the initial position during the collection process on the original copper wire. But for Sample-A, the droplets move towards to the more hydrophilic area gradually and coalesce into a bigger one during the collection process. The water transportation length $(L)$ reaches $1 \mathrm{~cm}$. The scales are $2 \mathrm{~mm}$. (c) Comparison of water collection abilities between original copper wire and Sample-A. Obviously, the copper wire with a multi-gradient displays a more efficient water collection and a larger hanging ability. process, the gradient copper wire displays a more efficient water collection and a larger hanging ability, which is mainly due to the fact that the additional driving forces from the wettable gradient and Laplace pressure gradient could induce directional movement of tiny water drops to reveal the original collection points for a new cycle of collection. ${ }^{19,23-26}$

\section{Conclusions}

In conclusion, in order to achieve longer transportation of a water droplet on a wire, we applied the gradient anodic oxidation method to fabricate a wettable gradient and Laplace pressure gradient simultaneously on a copper wire. By the cooperation of the wettable gradient and the Laplace pressure gradient, we realized the controlled movement of a droplet on a wire. The movement distance of a droplet can be adjusted between 0 and $4.5 \mathrm{~mm}$ for target transportation via the initial position and/or the magnitude of the wettable gradient. More interesting, the copper wire with a multi-gradient displays more efficient water collection and a larger hanging ability. This study is helpful in designing smart materials for developing applications in microfluidic devices and fog-water collection. ${ }^{25,26}$

\section{Acknowledgements}

This work was supported by the National Key Basic Research Program of China (2013CB933000), the National Natural Science Foundation of China (21673015), and Fundamental Research Funds for Central Universities (YWF-15-HHXY-017, YWF-16-JCTD-A-01) and Aeronautical Science Foundation of China (2015ZF51060).

\section{Notes and references}

1 Y. H. Liu, M. Andrew, J. Li, M. J. Yeomans and Z. K. Wang, Nat. Commun., 2015, 6, 10034.

2 F. Schellenberger, N. Encinas, D. Vollmer and H. J. Butt, Phys. Rev. Lett., 2016, 116, 096101.

3 K. H. Chu, R. Xiao and E. N. Wang, Nat. Mater., 2010, 9, 413.

4 E. Ueda and P. A. Levkin, Adv. Mater., 2013, 25, 1234.

5 C. Duprat, S. Protiere, A. Y. Beebe and H. A. Stone, Nature, 2012, 482, 510.

6 M. J. Hancock, K. Sekeroglu and M. C. Demirel, Adv. Funct. Mater., 2012, 22, 2223.

7 J. Bico, B. Roman, L. Moulin and A. Boudaoud, Nature, 2004, 432, 690.

8 Q. B. Wang, X. Yao, H. Liu, D. Quéré and L. Jiang, Proc. Natl. Acad. Sci. U. S. A., 2015, 112, 9247.

9 E. Lorenceau and D. Quere, J. Fluid Mech., 2004, 510, 29.

10 C. J. Lv, C. Chen, Y. C. Chuang, F. G. Tseng, Y. J. Yin, F. Grey and Q. S. Zheng, Phys. Rev. Lett., 2014, 113, 026101.

11 C. Schlaich, C. L. Cuellar, L. Yu, K. Achazi, Q. Wei and R. Haag, ACS Appl. Mater. Interfaces, 2016, 8, 29117.

12 S. L. Feng, S. J. Wang, L. C. Gao, G. J. Li, Y. P. Hou and Y. M. Zheng, Angew. Chem., Int. Ed., 2014, 53, 6163.

13 Y. Chen, L. Wang, Y. Xue, L. Jiang and Y. M. Zheng, Sci. Rep., 2013, 3, 2927. 
14 K. Manabe, T. Matsubayashi, M. Tenjimbayashi, T. Moriya, Y. Tsuge, K. H. Kyung and S. Shiratori, ACS Nano, 2016, 10, 9387.

15 F. Xiao, S. J. Yuan, B. Liang, G. Q. Li, S. O. Pehkonen and T. J. Zhang, J. Mater. Chem. A, 2015, 3, 4374.

16 M. M. Sung, K. Sung, C. G. Kim, S. S. Lee and Y. Kim, J. Phys. Chem. B, 2000, 104, 2273.

17 X. F. Wu, H. Bai, J. X. Zhang, F. E. Chen and G. Q. Shi, J. Phys. Chem. B, 2005, 109, 22836.

18 R. N. Wenzel, J. Phys. Colloid Chem., 1948, 53, 1466.

19 A. Ghosh, R. Ganguly, T. M. Schutzius and C. M. Megaridis, Lab Chip, 2014, 14, 1538.

20 J. Ju, K. Xiao, X. Yao, B. Hao and L. Jiang, Adv. Mater., 2013, 25, 5937.
21 X. Heng, M. M. Xiang, Z. H. Lu and C. Luo, ACS Appl. Mater. Interfaces, 2014, 6, 8032.

22 T. Xu, Y. C. Lin, M. X. Zhang, W. W. Shi and Y. M. Zheng, ACS Nano, 2016, 10, 10681.

23 P. Lovass, M. Branicki, R. Toth, A. Braun, K. Suzuno, D. Ueyama and I. Lagzi, RSC Adv., 2015, 5, 48563.

24 C. S. Sharma, J. Combe, M. Giger, T. Emmerich and D. Poulikakos, ACS Nano, 2017, 11, 1673.

25 R. L. Agapov, J. B. Boreyko, D. P. Briggs, B. R. Srijanto, S. T. Retterer, C. P. Collier and N. V. Lavrik, ACS Nano, 2013, 8, 860.

26 K. Manabe, T. Matsubayashi, M. Tenjimbayashi, T. Moriya, Y. Tsuge, K. H. Kyung and S. Shiratori, ACS Nano, 2016, 10, 9387. 